\title{
ОСОБЕННОСТИ АНАМНЕЗА, СОСТОЯНИЯ СОМАТИЧЕСКОГО И ГИНЕКОЛОГИЧЕСКОГО ЗДОРОВЬЯ ЖЕНЩИН С МЕНОПАУЗАЛЬНЫМ МЕТАБОЛИЧЕСКИМ СИНДРОМОМ
}

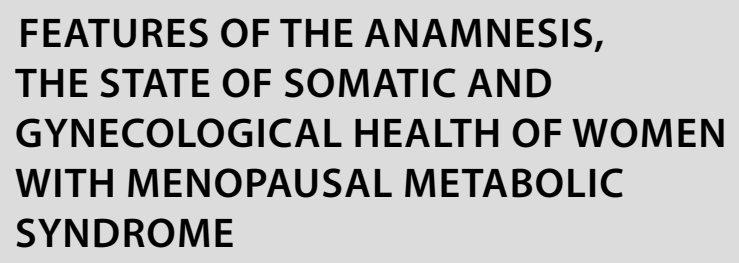

FEATURES OF THE ANAMNESIS, THE STATE OF SOMATIC AND GYNECOLOGICAL HEALTH OF WOMEN WITH MENOPAUSAL METABOLIC SYNDROME

Z. Shulukian

Summary. Introduction. In recent years, there has been an increased interest of researchers in the problems of the development of metabolic syndrome (MS) in menopausal women, due to the high risk of developing cardiovascular complications and type 2 diabetes mellitus. There are the following factors that contribute to the development of MS: genetic predisposition, sedentary lifestyle, low socio-economic status, peculiarities of eating behavior. Despite a fairly broad discussion of the development of MS, the issues of pathogenesis and conditions that contribute to the formation of MS continue to be discussed.

The aim of the study is to identify somatic and gynecological pathology that forms risk factors for the development of menopausal metabolic syndrome in women.

Methods. To achieve this goal, we examined 184 women aged 54.2 (0.5) years who were in menopause before 5 years. Of these, 87 were diagnosed with menopausal metabolic syndrome - they made up the main group. The comparison group consisted of 97 menopausal women without metabolic syndrome. The groups were comparable in terms of social status, level of education and nature of work activity.

Results and discussion. Risk factors for the development of menopausal metabolic syndrome were identified. It was found that of the gynecological diseases suffered, the most significant were SPMN and endometrial polyps. Of the somatic diseases suffered, the most important were diseases of the nervous system, in particular asthenovegetative syndrome, hypertension, pathology of the cardiovascular system. Obesity was less significant than the listed conditions.

Conclusion. Obesity, although it is one of the manifest forms of manifestation of the syndrome, does not play a leading role in the formation of pathology of the cardiovascular system or the development of type 2 diabetes, as the most threatening complications and extreme forms of MS. Menopausal symptoms, often accompanying menopause, should also be differentiated from the clinical manifestations of MS and those diseases that accompany or complicate the existing pathology during this period of a woman's life.
Шулукян Зепюр Егизаровна

Соискатель, ФГБОУ ВО «Самарский государственный медицинский университет» МЗ РФ zoyka_13@mail.ru

Аннотация. Введение. В последние годы возрос интерес исследователей к проблемам развития метаболического синдрома (МС) у женщин в менопаузе, обусловленный высоким риском развития сердечно-сосудистых осложнений и сахарным диабетом 2 типа. Выделяют следующие факторы, способствующие развитию МС: генетическая предрасположенность, малоподвижный образ жизни, низкий социально-экономический статус, особенности пищевого поведения. Несмотря на достаточно широкое 0бсуждение вопросов развития МС, вопросы патогенеза и состояний, способствующих формированию MC — продолжают дискутироваться.

Цель исследования — выделить соматическую и гинекологическую патологию, формирующую факторы риска развития менопаузального метаболического синдрома у женщин.

Методы. Для выполнения поставленной цели нами было обследовано 184 женщины в возрасте 54,2(0,5) лет, находившихся в менопаузе до 5 лет. Из них у 87 был диагностирован менопаузальный метаболический синдром — они составили основную группу. Группу сравнения составили 97 женщин в менопаузе без метаболического синдрома. Группы были сопоставимы по социальному положению, уровню образования и характеру трудовой деятельности.

Результаты и обсуждение. Были выделены факторы риска развития менопаузального метаболического синдрома. Установлено, что из перенесенных гинекологических заболеваний наиболее значимыми являлись СПМН и полипы эндометрия. Из перенесенных соматических заболеваний наибольшее значение имели заболевания нервной системы, в частности астено-вегетативный синдром, гипертония, патология сердечно-сосудистой системы. Ожирение было менее значимым, чем перечисленные состояния.

Заключение. Ожирение, хотя и является одной из манифестных форм проявления синдрома, не играет ведущей роли в формировании патологии сердечно-сосудистой системы или развития СД 2 типа, как наиболее грозных осложнений и крайних форм проявления МС. Климактерические симптомы, нередко сопровождающие менопаузу, также следует дифференцировать от клинических проявлений МС и тех заболеваний, которые сопутствуют или осложняют имеющуюся патологию в этот период жизни женщины.

Ключевые слова: менопаузальный метаболический синдром, факторы риска, ожирение, климактерический синдром. 
Keywords: menopausal metabolic syndrome, risk factors, obesity, menopausal syndrome.

\section{Введение}

B последние годы возрос интерес исследователей к проблемам развития метаболического синдрома (МС) у женщин в менопаузе, обусловленный высоким риском развития сердечно-сосудистых осложнений и сахарным диабетом 2 типа. По данным литературы факторами, способствующими развитию МС являются такие, как генетическая предрасположенность, малоподвижный образ жизни, низкий социально-экономический статус, особенности пищевого поведения $[1,6]$. Обсуждается снижение содержания эстрадиола и ожирение, как результат изменения кишечной микробиоты [2] Рассматриваются вопросы влияния дефицита кальциферола на развитие МС [3]. Вместе с тем, несмотря на достаточно широкое обсуждение вопросов развития МС и его проявлений таких, как дислипидемия, абдоминальное ожирение, гиперинсулинемия и инсулинорезистентность, нарушение в системе свертывания крови, вопросы патогенеза и состояний, способствующих формированию МС - продолжают дискутироваться $[4,5]$.

\section{Цель исслеАования}

В связи с изложенным, цель нашего исследования выделить соматическую и гинекологическую патологию, формирующую факторы риска развития менопаузального метаболического синдрома у женщин.

\section{Материал и методы}

Проведено обследование 184 женщин 54,2(0,5) лет, находившихся в менопаузе до 5 лет; 87 из них (основная группа) был выставлен MC, 97 женщин были без МС.

Критерии включения в групnы: менопауза 1-5 лет, отсутствие хронических экстрагенитальных заболеваний в стадии обострения, благоприятный онкологический анамнез, естественная менопауза.

Критерии исключения из групп: хирургическая менопауза, онкопатология любой локализации, обострение хронических соматических заболеваний.

Объем обследования: сбор анамнеза, общеклиническое обследование для определения гинекологического и соматического статуса.
Статистическая обработка данных: рассчитывалось среднее арифметическое (М) и стандартное отклонение (SD). Полученные качественные показатели в таблицах были представлены как в абсолютных значениях, так и в (\%) относительных величин. Оценка значимости различий полученных количественных данных, осуществлялась при помощи t- критерия Стьюдента для независимых выборок. Был проведен расчет отношения шансов (OR) развития метаболических нарушений (граница 95\% ДИ (CI)) Величина уровня значимости р была определена, как критическая при значениях $<0,05$.

\section{Результаты}

Нами были проанализированы анамнестические данные женщин, вошедших в группы сравнения, характеризующие нарушения репродуктивного периода, возможно способствующие развитию в последующем метаболических нарушений.

Рассматривая репродуктивный анамнез, отметим, что на бесплодие (в том числе эндокринное) в анамнезе не указывала ни одна женщина. Общее число беременностей, приходящихся на 1 пациентку основной группы, составило 4,6(0,7), а в группе сравнения $4,9(0,4),(p=0,710)$. Статистически значимые различия были получены только по числу поздних выкидышей в основной группе на 1 женщину число поздних выкидышей составило 0,04(0,001), в группе сравнения 0,03 $(0,001),(p<0,001)$. По указаниям на число естественных и оперативных родов, абортов, ранних выкидышей и внематочных беременностей, методов используемой в течение жизни контрацепции - статистически значимых различий в группах получено не было.

Рассматривая структуру перенесенной гинекологической патологии, отметим, что на первом месте в основной группе были такие состояния, как синдром предменструального напряжения (СПМН), на который указали 74,7\% пациенток, на втором месте были воспалительные заболевания шейки матки - 56,3\% женщин, далее следовали гиперпластические процессы.

На миому матки, развившуюся в репродуктивном периоде, указали 33,3\% женщин основной группы, на гиперпластические процессы эндометрия - 21,8\% пациенток. Отметим, что в группе сравнения аналогич- 
Таблица 1. Распределение женщин сравниваемых групп по жалобам в период обследования

\begin{tabular}{|c|c|c|c|}
\hline \multirow[t]{2}{*}{ Показатель } & $\begin{array}{l}\text { Основная группа } \\
(\mathrm{n}=87)\end{array}$ & $\begin{array}{l}\text { Группа сравнения } \\
(\mathbf{n = 9 7 )}\end{array}$ & \multirow[t]{2}{*}{$\mathbf{P}$} \\
\hline & \multicolumn{2}{|c|}{ Число женщин в группе —\% } & \\
\hline $\begin{array}{l}\text { Резкое повышение массы тела (более } \\
10 \text { кг в год) }\end{array}$ & \begin{tabular}{|l|}
78 \\
$89,7(3,3)$ \\
\end{tabular} & \begin{tabular}{|l}
2 \\
$2,1(1,5)$ \\
\end{tabular} & 0,000 \\
\hline Нарушения сна & $\begin{array}{l}52 \\
59,8(5,3) \\
\end{array}$ & $\begin{array}{l}18 \\
18,6(4,0) \\
\end{array}$ & 0,000 \\
\hline Утомляемость & $\begin{array}{l}72 \\
82,7(4,1)\end{array}$ & $\begin{array}{l}29 \\
30,0(4,7)\end{array}$ & 0,000 \\
\hline Апатия & $\begin{array}{l}11 \\
12,6(3,6)\end{array}$ & $\begin{array}{l}11 \\
11,3(3,2)\end{array}$ & 0,787 \\
\hline Потливость & $\begin{array}{l}76 \\
87,4(3,6) \\
\end{array}$ & $\begin{array}{l}16 \\
16,5(3,8) \\
\end{array}$ & 0,000 \\
\hline Запоры & $\begin{array}{l}27 \\
31,0(5,0)\end{array}$ & \begin{tabular}{|l}
8 \\
$8,2(2,8)$
\end{tabular} & 0,0001 \\
\hline Приступы тахикардии & $\begin{array}{l}77 \\
88,5(3,4)\end{array}$ & \begin{tabular}{|l}
21 \\
$21,6(4,2)$
\end{tabular} & 0,000 \\
\hline Повышение АД & $\begin{array}{l}62 \\
71,3(4,9)\end{array}$ & $\begin{array}{l}4 \\
4,1(2,0)\end{array}$ & 0,000 \\
\hline
\end{tabular}

Примечание: $\mathrm{p}$ - показатель статистической значимости различий в сравниваемых группах

Таблица 2. Распределение женщин сравниваемых групп по гинекологическому статусу

\begin{tabular}{|c|c|c|c|c|}
\hline \multirow[t]{2}{*}{ Показатель } & \multirow[t]{2}{*}{ Код МКБ-Х } & $\begin{array}{l}\text { Основная группа } \\
(\mathrm{n}=87)\end{array}$ & $\begin{array}{l}\text { Группа сравнения } \\
(\mathrm{n}=97)\end{array}$ & \multirow[t]{2}{*}{$\mathbf{P}$} \\
\hline & & \multicolumn{2}{|c|}{ Число женщин в группе —\% } & \\
\hline Миома матки & D25 & $\begin{array}{l}30 \\
34,4(5,1)\end{array}$ & \begin{tabular}{|l|}
33 \\
$34,0(4,8)$
\end{tabular} & 0,954 \\
\hline Воспалительные заболевания шейки матки & N86 & $\begin{array}{l}47 \\
54,0(5,3)\end{array}$ & $\begin{array}{l}44 \\
45,4(5,1)\end{array}$ & 0,243 \\
\hline Пролапс гениталий & N81 & $\begin{array}{l}28 \\
32,2(5,0) \\
\end{array}$ & $\begin{array}{l}31 \\
31,9(4,8) \\
\end{array}$ & 0,965 \\
\hline
\end{tabular}

Примечание: $\mathrm{p}$ - показатель статистической значимости различий в сравниваемых группах

ные показатели составили $22,7 \%, 45,4 \%, 34,0 \%$ и 2,1\%, соответственно.

Отметим, что статистически значимые различия были получены по таким заболеваниям, как СПМН $(p<0,001)$ и полипы эндометрия $(p<0,001)$. По остальным нозологическим формам гинекологической патологии статистически значимых различий выявлено не было.

Анализ перенесенных экстрагенитальных заболеваний, указывает на большое число патологии нервной системы, особенно таких ее проявлений, как остеохондроз различных отделов позвоночника и астено-невротический синдром- в основной группе таких пациенток было 71,2\%, в группе сравнения 48,5\% ( $<<0,001)$. На втором по частоте месте были указания на заболевания молочных желез, на фиброзно-кистозную мастопа- тию указывали 56,3\% женщин основной группы и 26,8\% женщин группы сравнения $(p<0,001)$. Далее следовали указания на заболевания сердечно-сосудистой системы, в частности, на варикозную болезнь - 47,1\% и12,4\%, соответственно ( $<<0,001)$. На гипертонию, развившуюся после 35 лет, указали 44,8\% пациентки основной группы и только 4,1\% в группе сравнения $(p<0,001)$. Сахарным диабетом 2 типа страдало 16,1\% женщин основной группы и 3,1\% группы сравнения $(p=0,003)$. Кроме того, имелись указания на патологию щитовидной железы (гипотиреоз), по поводу которого получали лечение $9,2 \%$ женщин основной группы. В группе сравнения таких пациенток не было.

Далее нами было оценено состояние здоровья женщин в период проведения исследования. Жалобы, с которыми женщины обратились за медицинской помощью, отражены в табл. 1 
Таблица 3. Распределение женщин сравниваемых групп по соматическому статусу

\begin{tabular}{|c|c|c|c|c|}
\hline \multirow[t]{2}{*}{ Показатель } & \multirow[t]{2}{*}{ Код МКБ-Х } & $\begin{array}{l}\text { Основная группа } \\
(\mathrm{n}=87)\end{array}$ & $\begin{array}{l}\text { Группа сравнения } \\
(\mathrm{n}=97)\end{array}$ & \multirow[t]{2}{*}{$\mathbf{P}$} \\
\hline & & \multicolumn{2}{|c|}{ Число женщин в группе —\% } & \\
\hline Гипертония & 111.9 & \begin{tabular}{|l|}
57 \\
$65,5(5,1)$ \\
\end{tabular} & \begin{tabular}{|l}
4 \\
$4,1(2,0)$ \\
\end{tabular} & 0,000 \\
\hline НАДЖБ & K76.0 & $\begin{array}{l}23 \\
26,4(4,7)\end{array}$ & $\begin{array}{l}1 \\
1,0(1,0) \\
\end{array}$ & 0,000 \\
\hline Нарушенная толерантность к глюкозе & R73.0 & $\begin{array}{l}69 \\
79,3(4,4) \\
\end{array}$ & $\begin{array}{l}3 \\
3,1(1,8) \\
\end{array}$ & 0,000 \\
\hline Сахарный диабет 2 типа & E11 & \begin{tabular}{|l|}
18 \\
$20,7(4,4)$
\end{tabular} & 0 & 0,000 \\
\hline Бронхиальная астма & $J 45$ & \begin{tabular}{|l|}
8 \\
$9,2(3,1)$
\end{tabular} & 0 & 0,003 \\
\hline Гипотиреоз & E01 & \begin{tabular}{|l|}
39 \\
$44,8(5,3)$
\end{tabular} & $\begin{array}{ll}2 \\
2,1(1,5)\end{array}$ & 0,000 \\
\hline Гипертиреоз & E05 & \begin{tabular}{|l|}
1 \\
$1,1(1,1)$
\end{tabular} & $\begin{array}{l}1 \\
1,0(1,0)\end{array}$ & 0,946 \\
\hline Желчекаменная болезнь & K80 & \begin{tabular}{|l|}
21 \\
$24,1(4,6)$
\end{tabular} & $\begin{array}{l}9 \\
9,2(2,9)\end{array}$ & 0,006 \\
\hline $\begin{array}{l}\text { Ожирение различной степени } \\
\text { выраженности }\end{array}$ & E66.0 & \begin{tabular}{|l|}
60 \\
$68,9(5,0)$
\end{tabular} & \begin{tabular}{|l|}
42 \\
$43,2(5,1)$
\end{tabular} & 0,0004 \\
\hline Хронический цистит & N30 & \begin{tabular}{|l|}
4 \\
$4,6(2,3)$
\end{tabular} & \begin{tabular}{|l|}
3 \\
$3,1(1,8)$
\end{tabular} & 0,608 \\
\hline Ревматоидный артрит & M06.0 & \begin{tabular}{|l|}
11 \\
$12,6(3,6)$
\end{tabular} & $\begin{array}{l}3 \\
3,1(1,8)\end{array}$ & 0,019 \\
\hline Ишемическая болезнь сердца & 125 & \begin{tabular}{|l|}
6 \\
$6,9(2,7)$ \\
\end{tabular} & 0 & 0,011 \\
\hline Остеохондроз позвоночника & M42 & \begin{tabular}{|l|}
71 \\
$81,6(4,2)$
\end{tabular} & \begin{tabular}{|l|}
52 \\
$53,6(5,1)$
\end{tabular} & $<0,001$ \\
\hline
\end{tabular}

Примечание: $\mathrm{p}$ - показатель статистической значимости различий в сравниваемых группах

Анализируя предъявляемые жалобы женщин основной группы, отметим, что наиболее часто пациентки указывали на резкое повышение массы тела, начавшееся за 1-2 года до наступления менопаузы - 89,7\%, приступы тахикардии 88,5\%, потливость - 87,4\%, утомляемость - 82,7\% и повышение АД - 71,3\%. Женщины группы сравнения наиболее часто жаловались на общую слабость - 30,0\%, приступы тахикардии 21,6\% и нарушения сна 18,6\%. Отметим, что по всем показателям между группами имелись статистически значимые различия $(p<0,001)$.

Что касается гинекологического статуса женщин, в момент включения в группы, то эти данные отражены в табл. 2.

В период обследования у 54\% пациенток основной группы и у $45,4 \%$ женщин группы сравнения $(p=0,243)$ наиболее часто выявлялись воспалительные заболевания шейки матки, связанные с атрофическими процессами слизистой (как следствие менопаузы). На втором месте по частоте была выявлена миома матки - 34,4\% пациенток основной группы и 34,0\% группы сравнения
( $p=0,954)$. Укажем, что у всех пациенток миома матки оценивалась, как бессимптомная и эти пациентки не нуждались в терапевтических мерах или хирургическом лечении миомы матки. У трети женщин в каждой группе имели проляпс половых органом в разной стадии выраженности - таких пациенток в основной группе было 32,2\%, в группе сравнения - 31,9\% ( $p=0,965)$.

Далее нами был проанализированы данные общего состояния здоровья обследуемых пациенток (табл. 3).

Рассматривая сопутствующую экстрагенитальную патологию, отметим, что большинство патологических состояний укладывались в комплекс метаболических расстройств. В основной группе у всех женщин диагностировались нарушения толерантности к глюкозе, а у каждой 5 пациентки был выявлен сахарный диабет 2 типа $(20,7 \%)$. Кроме того, в основной группе у $65,5 \%$ женщин регистрировалось повышение артериального давления, которое требовало систематической фармакологической коррекции. Гипотиреоз был выявлен у 44,8\% пациенток. Неалкогольная жировая дистрофия печени (НАЖДП) была установлена у 26,4\% женщин основной 
Таблица 4. Факторы риска развития МС у женщин в менопаузе

\begin{tabular}{|c|c|c|c|}
\hline $\begin{array}{l}\text { Фактор риска (шанс найти фактор риска в основной группе - } \\
\text { шанс найти фактор риска в группе сравнения) }\end{array}$ & Код МКБ-Х & OR(S) & $\mathbf{C i}$ \\
\hline \multicolumn{4}{|l|}{ Анамнез гинекологической патологии } \\
\hline Полип эндометрия (0,235-0,021) & N84 & $11,176(0,767)$ & $2,487-50,220$ \\
\hline Синдром предменструального напряжения $(2,955-0,293)$ & N94.3 & $10,072(0,346)$ & $5,113-19,840$ \\
\hline \multicolumn{4}{|l|}{ Анамнез соматической патологии } \\
\hline Заболевания системы пищеварения $(0,338-0,128)$ & K00-K93 & $2,646(0,404)$ & $1,198-5,844$ \\
\hline Заболевания сердечно-сосудистой системы $(0,891-0,141)$ & $100-199$ & $6,313(0,376)$ & $3,023-13,187$ \\
\hline Гипертония $(0,813-0,043)$ & 111.9 & $18,891(0,554)$ & $6,374-55,983$ \\
\hline $\begin{array}{l}\text { Заболевания нервной системы (астеноневротический синдром, } \\
\text { остеохондроз позвоночника) }(2,480-0,940)\end{array}$ & G55.1 & $2,638(0,312)$ & $1,431-4,864$ \\
\hline Патология молочных желез $(1,289-0,366)$ & N60.1 & $3,521(0,315)$ & $1,899-6,530$ \\
\hline \multicolumn{4}{|l|}{ Сопутствующая соматическая патология } \\
\hline Гипертония $(1,900-0,043)$ & 111.9 & $44,175(0,558)$ & $14,791-131,935$ \\
\hline НАДЖБ $(0,359-0,010)$ & K76.0 & $34,5(1,034)$ & $4,545-216,895$ \\
\hline Нарушенная толерантность к глюкозе $(3,833-0,032)$ & R73.0 & $120,111(0,643)$ & $34,031-423,929$ \\
\hline Гипотиреоз $(0,813-0,021)$ & E01 & $38,594(0,746)$ & $8,938-166,649$ \\
\hline Желчекаменная болезнь $(0,318-0,102)$ & K80 & $3,111(0,430)$ & $1,338-7,232$ \\
\hline Ожирение различной степени выраженности $(2,222-0,764)$ & E66.0 & $2,910(0,309)$ & $1,587-5,336$ \\
\hline Ревматоидный артрит)0,145-0,032) & M06.0 & $4,535(0,669)$ & $1,221-16,840$ \\
\hline
\end{tabular}

группы. Отметим, что различные стадии ожирения регистрировались у 68,9\% женщин, а у остальных $31,1 \%$ масса тела была повышенной. Перечисленные состояния формировали клинику метаболического синдрома: ожирение, нарушение липидного и углеводного обмена, сопровождающегося изменением состояния сердечно-сосудистой системы. Что касается женщин группы сравнения, то, несмотря на достаточно высокий удельный вес женщин с ожирением 1 и 2 степени (43,2\%), остальных проявлений метаболического синдрома у них выявлено не было; 53,6\% страдали остеохондрозом (что могло расцениваться, как следствие снижения костной массы в сочетании с высоким весом) и у 9,2\% пациенток была диагностирована желчекаменная болезнь, что объяснялось, по - видимому, пристрастиями пищевого поведения и в части случаев особенностями анатомического строения желчного пузыря. При этом поражения сердечно-сосудистой системы и гиперинсулинемии у пациенток группы сравнения установлено не было.

Мы рассчитали факторы риска пациенток по развитию МС. Для расчета факторов риска использовали методику отношения шансов OR (Ci) с определением доверительного интервала. В таблицу включали значения OR больше 1. Результаты выделенных факторов риска мы вывели в отдельную таблицу, ранжировав их в порядке убывания рисков (табл. 4)

Анализируя полученные результаты расчета отношения шансов у женщин в менопаузе, мы выделили основные патологии, формирующие предпосылки к раз- витию метаболических нарушений. Из перенесенных гинекологических заболеваний наиболее значимыми являлись такие, как СПМН и полипы эндометрия. У таких пациенток шанс развития метаболических нарушений был в 10 раз выше, чем у женщин, не имеющих в анамнезе подобных патологий.

Рассматривая перенесенную экстрагенитальную патологию отметим, что наибольшее значение имели такие состояния, как заболевания нервной системы, в частности астено-вегетативный синдром, у таких пациенток шанс развития МС в менопаузе был в 26 раз выше; на втором месте по значимости была гипертония, шанс развития МС у этих пациенток возрастал в 19 раз, на третьем месте были женщины с патологией сердечно-сосудистой системы - их шансы на развитие МС возрастали в 8 раз.

В менопаузе наибольшее значение имели такие состояния, как нарушения толерантности к глюкозе шанс развития МС у таких женщин повышался в 120 раз, с гипертонией - в 44 раза, с гипотиреозом - в 39 раз и НАДЖБ - в 36 раз. Что касается ожирения, то этот фактор повышал шанс развития МС только в 3 раза.

\section{Зак^ючение}

Таким образом, оценивая медико-социальные показатели женщин сравниваемых групп, укажем, что ожирение, хотя и является одной из манифестных форм проявления синдрома, не играет ведущей роли в фор- 
мировании патологии сердечно-сосудистой системы или развития СД 2 типа, как наиболее грозных осложнений и крайних форм проявления МС. Климактерические симптомы, нередко сопровождающие менопаузу, также следует дифференцировать от клинических проявлений МС и тех заболеваний, которые сопутствуют или осложняют имеющуюся патологию в этот период жизни женщины.

\section{ЛИТЕРАТУРА}

1. Raczkiewicz D., Owoc A., Wierzbińska-Stępniak A., Bojar I. Metabolic syndrome in peri- and postmenopausal women performing intellectual work. Annals of Agricultural and Environmental Medicine. 2018;25(4):610-615. D0I: 10.26444/aaem/74451

2. Gurka M.J., Vishnu A., Santen R.J., DeBoer M.D. Progression of Metabolic Syndrome Severity During the Menopausal Transition. Journal of the American Heart Association. 2016;5(8):003609. DOI: 10.1161/JAHA.116.003609

3. Schmitt E.B., Nahas-Neto J., Bueloni-Dias F. et al. Vitamin D deficiency is associated with metabolic syndrome in postmenopausal women. Maturitas. 2018;107:97-102. D0I: 10.1016/j.maturitas.2017.10.011

4. Baker J.M., Al-Nakkash L., Herbst-Kralovetz M.M. Estrogen-gut microbiome axis: Physiological and clinical implications. Maturitas. 2017;103:45-53. D0I: 10.1016/j.maturitas.2017.06.025

5. Эседова А.Э., Уруджева Н.Г., Ильина И.Ю. Менопаузальный метаболический синдром и риски назначения менопаузальной гормональной терапии. Пути решения. РМЖ. Мать и дитя. 2020; 4: 260-266. Режим доступа: https://www.rmj.ru/articles/ginekologiya/Menopauzalynyy_metabolicheskiy_ sindrom_i_riski_naznacheniya_menopauzalynoy_gormonalynoy_terapii_Puti_resheniya/

6. Рекомендации по ведению больных с метаболическим синдромом. Клинические рекомендации. М.; 2013. Режим доступа: https://mzdrav.rk.gov.ru/ file/mzdrav_18042014_Klinicheskie_rekomendacii_Metabolicheskij_sindrom.pdf

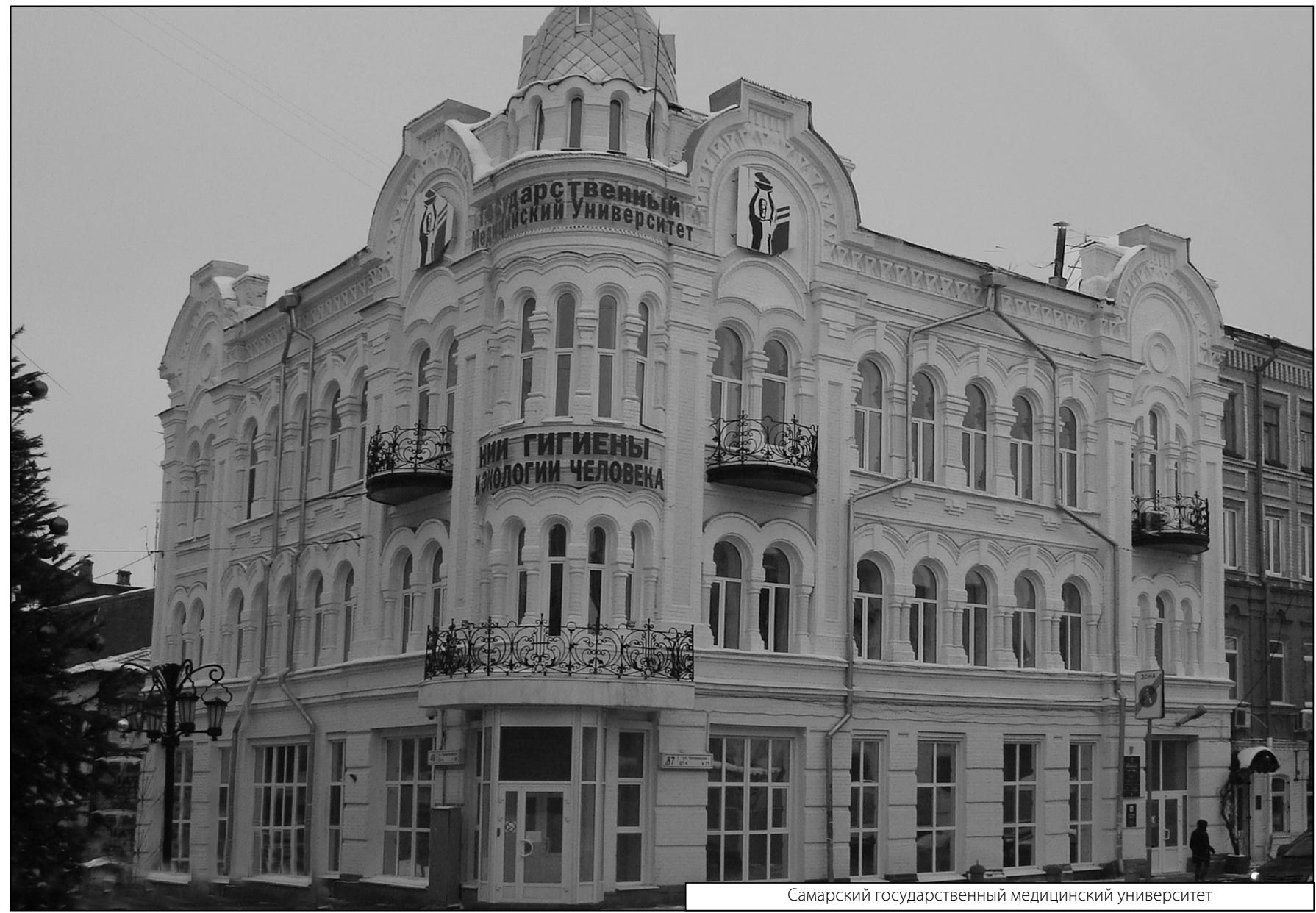

